\title{
Frozen elephant trunk: reflections of the UK Aortic Group
}

\author{
Aung Oo ${ }^{1}$, Mark L. Field ${ }^{2}$ \\ ${ }^{1}$ Clinical Lead for Aortovascular Surgery, The Barts Heart Centre, London, UK; ${ }^{2}$ Clinical Lead for Cardiac Surgery, Liverpool Heart and Chest \\ Hospital, Liverpool, UK \\ Correspondence to: Mark L. Field, FRCS (C-Th), DPhil (Oxon). Clinical Lead Cardiac Surgery, Liverpool Heart and Chest Hospital, Liverpool, UK. \\ Email: Mark.Field@lhch.nhs.uk.
}

Submitted Jan 17, 2020. Accepted for publication Jan 28, 2020.

doi: $10.21037 /$ acs.2020.02.04

View this article at: http://dx.doi.org/10.21037/acs.2020.02.04

The frozen elephant trunk (FET) has undoubtedly saved many lives, offering solutions without which interventions would have been challenging, if not impossible. Originally designed to treat aortic arch and proximal descending thoracic aortic pathology as a one stage procedure, uses have evolved into providing a platform for second-stage open and thoracic aortic endovascular repair (TEVAR) procedures, as well as in acute and chronic dissection, positively modifying the natural history of the false lumen. More nuanced zone zero deployment with de-branching has enabled treatment of the most extreme of aortic arch pathologies. However, FET has proven most versatile in acute type A aortic dissection by managing the false lumen in the proximal descending thoracic aorta (DTA), a segment known to evolve quickly following conventional surgery. The "UK-Aortic Group", a collaborative group of aorto-vascular surgeons, have reviewed the outcomes from use of the FET in acute type A aortic dissection (1) within high volume UK centres, reporting favorable outcomes for mortality (12\%), temporary/permanent neurological impairment $(17 \%)$, renal replacement therapy $(20 \%)$ and permanent paraplegia $(0 \%)$; remarkably better than the UK national average for all operations across all centres. As the mortality outcome for hemiarch versus total arch with FET in acute type A approaches equipoise, there is increasing interest in a randomised controlled trial with both in-patient outcomes and a composite 3 -year outcome of survival, aortic-related morbidity and re-intervention.

Several challenges remain for the FET however, in both the elective and emergency setting. The FET has a complication profile which is different to conventional elephant trunk (CET) and, in relative terms, is not an entirely benign addition to an arch replacement. There are additional risks of paraplegia, endoleaks, endoluminal clots and adverse remodelling of distal aortic disease, which are not seen in CET. Use of the device has become increasingly liberal, including in borderline disease, complex pathology and outside the context of a multi-disciplinary team or agreed plan for distal intervention. These complications may be life threatening and it is incumbent on all physicians to ensure that due diligence in discussion, planning and consent is in place. Centres should have considered how to manage complications such as paraplegia including arrangements for cerebrospinal fluid (CSF) drainage, motor evoked potentials (MEP) monitoring and complex secondary endovascular intervention if required. Protocols for follow-up are essential and second-stage procedures must be religiously planned for. The culture should not be of simple deployment but of institutional governance and systems in place to support the entire peri-operative pathway over the lifetime of the patient.

Technological innovations in the future will undoubtedly need to focus on rapid access to bespoke FET devices, not only in terms of length but also diameter, radial force and branch configuration. It should be acknowledged that several devices are available worldwide, including the Terumo-Aortic, Jotec and several bespoke devices constructed in China, India and South East Asia. Tight governance is required to care for the product. Without this, there is an inevitable risk of poor outcomes and reputational compromise.

\section{Acknowledgments}

None. 


\section{Footnote}

Conflicts of Interest: The authors have no conflicts of interest to declare.

Open Access Statement: This is an Open Access article distributed in accordance with the Creative Commons Attribution-NonCommercial-NoDerivs 4.0 International License (CC BY-NC-ND 4.0), which permits the noncommercial replication and distribution of the article with the strict proviso that no changes or edits are made and the

Cite this article as: Oo A, Field ML. Frozen elephant trunk: reflections of the UK Aortic Group. Ann Cardiothorac Surg 2020;9(3):228-229. doi: 10.21037/acs.2020.02.04 original work is properly cited (including links to both the formal publication through the relevant DOI and the license). See: https://creativecommons.org/licenses/by-nc-nd/4.0/.

\section{References}

1. Mariscalco G, Bilal H, Catarino P, et al. Reflection From UK Aortic Group: Frozen Elephant Trunk Technique as Optimal Solution in Type A Acute Aortic Dissection. Semin Thorac Cardiovasc Surg 2019;31:686-90. 